\title{
Adenomas Colorretais: Fatores de Risco Associados à Displasia de Alto Grau
}

\author{
Colorectal Adenomas: Risk Factors for High-Grade Dysplasia
}

\author{
JULIASCHMIDT SILVA ${ }^{1}$; AFONSO CALIL MURY MALLMANNN ${ }^{2,3}$; RUYTAKASHI KOSHIMIZU' ${ }^{2,3}$; DANIELA \\ CERQUEIRAKOPPE ${ }^{2}$; FABÍOLAELIZABETE SAVARIS ${ }^{4}$; LUCIANO PINTODE CARVALHO $^{2,3,5}$
}

\author{
1. Acadêmica do XII semestre do Curso de Medicina da Universidade Luterana do Brasil (ULBRA) Canoas/RS; \\ 2. Médico(a) do Serviço de Coloproctogia do Hospital Nossa Senhora da Conceição de Porto Alegre/RS; ${ }^{3}$ Titular pela \\ Sociedade Brasileira de Coloproctologia; ${ }^{4}$ Acadêmica do XI semestre do Curso de Medicina da Universidade Luterana \\ do Brasil (ULBRA) Canoas/RS; '5. Professor Adjunto da Universidade Luterana do Brasil (ULBRA) Canoas/RS.
}

\begin{abstract}
SILVA JS; MALLMANN ACM; KOSHIMIZU RT; KOPE DC; SAVARIS FE; CARVALHO LP. Adenomas Colorretais: Fatores de Risco Associados à Displasia de Alto Grau. Rev bras Coloproct, 2009;29(2): 209-215.

RESUMO: Fundamentos: O estudo dos pólipos, em especial os adenomatosos, é relevante devido à correlação direta com o carcinoma colorretal. Objetivo: Analisar quais são os fatores de risco para uma displasia de alto grau do pólipo adenomatoso retirados endoscopicamente. Métodos: Avaliamos todas as colonoscopias realizadas pelo Serviço de Coloproctologia no período de janeiro de 2003 a dezembro de 2006. Foram incluídos os pacientes com diagnóstico de pólipos adenomatosos. Analisamos a existência da relação entre adenomas com displasia de alto grau e os fatores associados dos pacientes e pólipos. Resultados: Foram realizados 1821 exames e encontramos 208 pacientes com 326 adenomas. 51,4\% do sexo masculino e 69,2\% tinham um único adenoma. Foram encontrados 28 pacientes $(13,5 \%)$ com, ao total, 34 adenomas $(10,4 \%)$, com displasia de alto grau. Entre os adenomas com displasia de alto grau, $64,7 \%$ eram $=1 \mathrm{~cm}, 61,8 \%$ eram sésseis, $29,4 \%$ vilosos e $70,6 \%$ estavam localizados no cólon esquerdo. Comparando as características do grupo dos adenomas com displasia de baixo grau com o de alto grau, foi estatisticamente significativo o tamanho $=1 \mathrm{~cm}$ e o componente viloso $(P<0,001)$. Conclusão: Adenomas $=1 \mathrm{~cm}$ e a predominância do componente viloso foram considerados fatores de risco independentes para displasia de alto grau.
\end{abstract}

Descritores: Adenoma; Adenocarcinoma; Pólipos; Neoplasias colorretais; Colonoscopia.

\section{INTRODUÇÃO}

O câncer colorretal (CCR) é uma doença muito prevalente com altas taxas de mortalidade. É a terceira causa mais comum de câncer no mundo, em ambos os sexos, e a segunda causa nos países desenvolvidos. Em 2008, estima-se que 148.810 pessoas irão ser diagnosticadas com CCR e 49.960 morrerão desta doença nos Estados Unidos ${ }^{(1)}$. O número de casos novos de CCR estimados para o Brasil, no ano de 2008, é de 12.490 casos em homens e de 14.500 em mulheres. Na região Sul do Brasil, sem considerar os tumores de pele não-melanoma, o câncer colorretal ocupa a quarta posição para os homens e a terceira para as mulheres ${ }^{(2)}$.

A maioria dos CCR cresce lentamente a partir de pólipos adenomatosos através da sequência conhecida como adenoma-carcinoma em combinação com alterações genéticas e mudanças ambientais ${ }^{(3)}$. Há evidência que a evolução dos adenomas com displasia leve para carcinoma invasivo se desenvolva em, aproximadamente, $10 \operatorname{anos}^{(4)}$.

Embora $30-50 \%$ das pessoas desenvolvam durante a sua vida pólipos adenomatosos, apenas cerca de 3-6\% da população é diagnosticada com $\mathrm{CCR}^{(5,6)}$. 
A relevância do estudo dos pólipos, em especial os adenomatosos, é a sua correlação direta com o CCR. A remoção dos adenomas pode prevenir o CCR, assim como o diagnóstico precoce pode reduzir a mortalidade $^{(7,8,9,10,11)}$.

Os adenomas são classificados histologicamente conforme a participação do componente tubular e viloso na sua constituição, e também conforme o seu grau de displasia, que pode ser de baixo ou alto $\operatorname{grau}^{(12)}$.

Atualmente, a displasia de alto grau é usada como um marcador de potencial maligno, logo, o conhecimento das características destes pólipos poderia ajudar a elucidar os fatores associados com o desenvolvimento do adenoma para o adenocarcinoma ${ }^{(13)}$.

De acordo com o National Polyp Study, um centro de referência de pesquisas de pólipos, os fatores mais importantes relacionados aos adenomas com displasia de alto grau são tamanho $=1 \mathrm{~cm}$, idade avançada e a presença de componente viloso ${ }^{(14)}$. Portanto, a identificação dos fatores de risco para os adenomas com displasia de alto grau pode contribuir de maneira significativa para o tratamento e seguimento dos pacientes com pólipos adenomatosos.

O objetivo deste estudo é analisar quais são os fatores de risco para uma displasia de alto grau do pólipo adenomatoso retirados endoscopicamente.

\section{PACIENTES E MÉTODOS}

Trata-se de um estudo analítico, longitudinal e retrospectivo com dados secundários de registro hospitalar. Neste trabalho foram analisados os laudos de colonoscopia, e os respectivos anátomo-patológicos dos pólipos retirados através de polipectomia endoscópica de pacientes submetidos ao exame no Serviço de Coloproctologia do Hospital Nossa Senhora da Conceição, em Porto Alegre, Rio Grande do Sul, no período de janeiro de 2003 a dezembro de 2006. Foi elaborado um banco de dados reunindo os laudos das colonoscopias e os resultados dos anátomopatotológicos dos adenomas. Durante quatro anos, foram realizados 1821 exames de colonoscopias e, entre estes, foram encontrados 208 pacientes com adenomas, sendo identificados, ao total, 326 pólipos adenomatosos.

Todos os pacientes com pólipos adenomatosos foram incluídos neste estudo. Foram excluídos aqueles pacientes diagnosticados com polipose adenomatosa familiar, pólipos que não eram adenomatosos, carcinoma invasivo, laudos e resultados de anátomo-patológicos incompletos.

Os pacientes foram avaliados quanto à idade, gênero, presença ou não de pólipos adenomatosos à colonoscopia e o número de pólipos. Os adenomas foram analisados quanto ao tamanho, à distribuição geográfica ao longo do intestino grosso, aparência macroscópica, características histológicas e o grau de displasia.

\section{Classificação dos adenomas}

Em nosso estudo, para análise estatística, agrupamos algumas variáveis em uma mesma categoria, baseado nos achados mais frequentes de fatores de risco para displasia de alto grau. O número de pólipos por paciente foi divido em dois grupos, um com menos de três pólipos e, outro, com três ou mais pólipos.

Dividimos o intestino grosso em três partes, a saber: a) o cólon direito, definido como o ceco, cólon ascendente e ângulo hepático; b) cólon transverso; c) o cólon esquerdo, incluindo o ângulo esplênico, cólon descendente, cólon sigmóide e o reto. Pólipos pediculados e subpediculados foram agrupados em um único grupo e separados dos sésseis. Dividimos os pólipos em dois grupos de acordo com o tamanho: $<1 \mathrm{~cm} \mathrm{e}=1 \mathrm{~cm}$.

A análise histológica dos pólipos removidos endoscopicamente foi realizada pelos patologistas de acordo com critérios unificados. A classificação histológica do adenoma e o grau de displasia foram baseados nos critérios do World Health Organization (WHO) que utiliza, para a classificação dos adenomas, a participação do componente viloso e tubular em sua constituição. Quando o componente viloso for menor que $25 \%$, este será considerado adenoma tubular e se maior que $75 \%$ é considerado adenoma viloso. Quando o componente viloso estiver entre $25 \%$ e $75 \%$ é classificado como túbulo-viloso.

A displasia dos adenomas foi classificada como baixo e alto grau baseado no grau de atipia celular e displasia tecidual. Displasia de baixo grau incluiu adenomas com displasia leve ou moderada, enquanto, displasia de alto grau incluiu displasias severas e, por alguns autores, carcinoma in situ. O diagnóstico de carcinoma invasivo, dentro de um adenoma, é feito somente se houver a invasão pelo carcinoma da muscular da mucosa através da submucosa. 


\section{Análise estatística}

Os dados dos pacientes e dos pólipos foram digitados em um único banco de dados criado no programa Microsoft Office Excel 2003 e posteriormente exportados para o programa SPSS v.14.0 para análise dos mesmos. Foram analisadas as variáveis quantitativas pela média e desvio padrão, e as variáveis categóricas pela frequência absoluta e frequência relativa percentual. Foram comparadas as variáveis quantitativas entre os grupos pelo teste t de Student para amostras independentes. Para as variáveis categóricas foi usado o teste de Qui-quadrado, teste de Qui-quadrado com correção de Yates ou teste Exato de Fisher. Foi considerado um nível de significância de 5\%.

\section{RESULTADOS}

Características dos pacientes e adenomas

Foram realizados 1.821 exames de colonoscopias e, entre estes, encontrados 208 pacientes com adenomas, sendo identificados ao total, 326 pólipos adenomatosos. A idade dos pacientes com adenomas variou de 16 a 98 anos, com a média de 63,5 $\pm 13,5$ anos. Entre os pacientes, 107 (51,4\%) homens e 101 $(48,6 \%)$ mulheres. As características dos pacientes submetidos à polipectomia estão apresentadas na Tabela 1.

Achados patológicos revelaram que 180 $(86,5 \%)$ pacientes tiveram displasia leve, enquanto no restante dos pacientes, $28(13,5 \%)$, foram diagnosticados com displasia de alto grau.

Entre as prevalências de pólipos nas diferentes categorias de idade houve diferença estatisticamente significativa $(P<0,001)$. Esta diferença foi localizada entre as categorias < 40, 40-49 anos e a categoria 70-79 anos e pode ser visualizada no Gráfico 1. Quando divididos em duas categorias, $<50 \mathrm{e}=50$ anos, também encontra-se uma diferença significativa $(P<0,001)$.

\section{Adenomas e suas respectivas displasias}

As características dos 208 pacientes e dos 326 adenomas ressecados são apresentadas nas Tabelas 2 e 3. Os pólipos adenomatosos foram divididos em duas categorias, displasia de alto grau e displasia de baixo grau. Os pacientes e as displasias dos adenomas foram comparados. A prevalência de displasia de alto grau foi semelhante entre os gêneros masculino e feminino. Entre os 326 adenomas retirados endos- copicamente encontrou-se um maior número de adenomas com displasia de alto grau com tamanho $=1$ $\mathrm{cm}(P<0,001)$. Adenomas vilosos foram mais frequentemente observados em displasia de alto grau $(29,4 \%)$ do que em displasia de baixo grau $(4,1 \%),(P$ $<0,001)$.

Tabela 1 - Características dos 208 pacientes e dos 326 adenomas ressecados por polipectomia.

\begin{tabular}{|c|c|c|}
\hline Variáveis & & \\
\hline Idade média \pm DP (anos) & & $5 \pm 13,5$ \\
\hline Gênero & & \\
\hline Masculino & 107 & $(51,4 \%)$ \\
\hline Feminino & 101 & $(48,6 \%)$ \\
\hline Número de adenomas por & & \\
\hline 1 & 144 & $(69,2 \%)$ \\
\hline 2 & 36 & $(17,3 \%)$ \\
\hline 3 & 14 & $(6,7 \%)$ \\
\hline$=4$ & 14 & $(6,7 \%)$ \\
\hline Localização & & \\
\hline Ceco & 19 & $(5,8 \%)$ \\
\hline Cólon ascendente & 39 & $(12 \%)$ \\
\hline Ângulo hepático & 22 & $(6,8 \%)$ \\
\hline Cólon transverso & 47 & $(14,5 \%)$ \\
\hline Ângulo esplênico & 7 & $(2,1 \%)$ \\
\hline Cólon descendente & 21 & $(6,4 \%)$ \\
\hline Cólon sigmóide & 122 & $(37,4 \%)$ \\
\hline Reto & 49 & $(15 \%)$ \\
\hline Aparência macroscópica & & \\
\hline Pediculado & 85 & $(26 \%)$ \\
\hline Subpediculado & 9 & $(2,8 \%)$ \\
\hline Séssil & 232 & $(71,2 \%)$ \\
\hline Histologia do adenoma & & \\
\hline Tubular & 251 & $(77 \%)$ \\
\hline Túbulo-viloso & 53 & $(16,3 \%)$ \\
\hline Viloso & 22 & $(6,7 \%)$ \\
\hline Tamanho em cm $(n=318)$ & & \\
\hline$<0,5$ & 175 & $(55 \%)$ \\
\hline $0,5-0,9$ & 57 & $(17,9 \%)$ \\
\hline$=1$ & 86 & $(27,1 \%)$ \\
\hline Tipo de displasia & & \\
\hline Alto grau & 34 & $(10,4 \%)$ \\
\hline Baixo/moderado grau & 292 & $(89,6 \%)$ \\
\hline
\end{tabular}




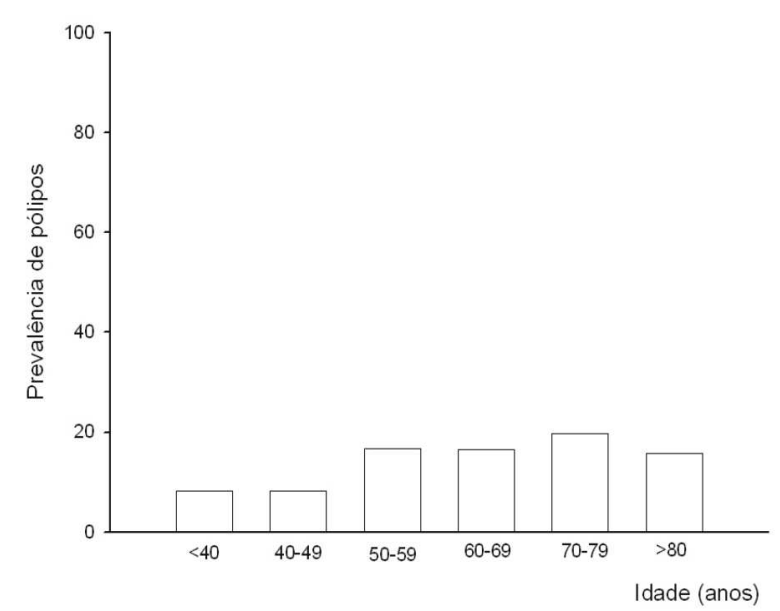

Gráfico 1 - Prevalência de pólipos adenomatosos por faixa etária dos pacientes.

\section{DISCUSSÃO}

O CCR é a terceira maior causa de morte por câncer no mundo inteiro ${ }^{(1)}$. A incidência desta neoplasia tem diminuído desde que o rastreamento e a vigilância para o câncer colorretal tornaram-se mais frequentes ${ }^{(7)}$.

Tem sido bem documentado que aproximadamente dois terços de todos os casos de CCR surgem a partir de adenomas através da sequência adenomacarcinoma. Para o terço restante, porém, o mecanismo de desenvolvimento ainda não foi bem estabelecido. Aproximadamente $40 \%$ da população ocidental desenvolverão adenomas, e somente uma pequena porcentagem irá progredir para o câncer colorretal ${ }^{(6)}$.

De acordo com o National Polyp Study, existem fortes evidências de que a progressão do adenoma

Tabela 2 - Características dos pacientes com adenomas ( $n=208)$.

\begin{tabular}{|c|c|c|c|}
\hline Variáveis & $\begin{array}{l}\text { Displasia de alto grau } \\
\qquad(n=28)\end{array}$ & $\begin{array}{l}\text { Displasia de baixo grau } \\
\qquad(n=180)\end{array}$ & Valor de $P$ \\
\hline Idade média \pm DP & $65,1+/-13,2$ & $63,2+/-13,6$ & 0,480 \\
\hline \multicolumn{4}{|l|}{ Gênero } \\
\hline Masculino & $(50 \%)$ & $(51,7 \%)$ & 0,999 \\
\hline Feminino & $(50 \%)$ & $(48,3 \%)$ & \\
\hline \multicolumn{4}{|l|}{ Número de pólipos } \\
\hline$<3$ & $(75 \%)$ & $(88,3 \%)$ & 0,072 \\
\hline$=3$ & $(25 \%)$ & $(11,7 \%)$ & \\
\hline
\end{tabular}

Tabela 3 - Características dos pólipos adenomatosos ( $n=326)$.

\begin{tabular}{|c|c|c|c|}
\hline Variáveis & $\begin{array}{c}\text { Displasia de alto grau } \\
(n=34)\end{array}$ & $\begin{array}{l}\text { Displasia de baixo grau } \\
(n=292)\end{array}$ & Valor de $P$ \\
\hline \multicolumn{4}{|l|}{ Localização } \\
\hline Cólon direito & $8 \quad(23,5 \%)$ & $72 \quad(24,6 \%)$ & 0,282 \\
\hline Transverso & $2 \quad(5,9 \%)$ & $45 \quad(15,4 \%)$ & \\
\hline Cólon esquerdo & $24 \quad(70,6 \%)$ & $175 \quad(60 \%)$ & \\
\hline \multicolumn{4}{|l|}{ Aparência macroscópica } \\
\hline Pediculado/subpediculado & $13 \quad(38,2 \%)$ & $81 \quad(27,7 \%)$ & 0,267 \\
\hline Séssil & $21 \quad(61,8 \%)$ & $211 \quad(72,3 \%)$ & \\
\hline \multicolumn{4}{|l|}{ Tamanho $(\mathrm{cm})(n=318)$} \\
\hline$<1$ & $12 \quad(35,3 \%)$ & $222 \quad(78,2 \%)$ & $<0,001$ \\
\hline$=1$ & $22 \quad(64,7 \%)$ & $62 \quad(21,8 \%)$ & \\
\hline \multicolumn{4}{|l|}{ Adenoma } \\
\hline Tubular & $10 \quad(29,4 \%)$ & $241 \quad(82,5 \%)$ & $<0,001$ \\
\hline Túbulo-viloso & $14 \quad(41,2 \%)$ & $39 \quad(13,4 \%)$ & \\
\hline Viloso & $10 \quad(29,4 \%)$ & $12 \quad(4,1 \%)$ & \\
\hline
\end{tabular}


para o adenocarcinoma pode ser reduzida através da prática atual de procura e remoção dos adenomas no cólon através da polipectomia endoscópica ${ }^{(15)}$. O adenoma com displasia de alto grau é considerado uma ponte patológica entre uma lesão benigna e o câncer invasivo $^{(14)}$

Em nosso estudo, foram realizadas 1821 colonoscopias, durante um período de quatro anos, e 326 adenomas foram retirados por polipectomia endoscópica e analisados quanto aos fatores de risco para displasia de alto grau.

Segundo o estudo de Kurome et al. ${ }^{(13)}$, tiveram como resultados o sexo feminino como um fator de risco para displasia de alto grau, contradizendo estudos anteriores que afirmavam ter o sexo masculino como fator de risco ${ }^{(16,17,18,19,20)}$. Dois estudos que analisaram apenas lesões ressecadas endoscopicamente (sem incluir câncer invasivo) indicaram que a prevalência da displasia de alto grau foi semelhante entre os sexos masculino e feminino ${ }^{(4,14)}$. Em nosso estudo, encontramos 28 pacientes $(13,5 \%)$ com adenomas com displasia de alto grau, e também não encontramos o gênero como um fator de risco independente.

Kurome et al. ${ }^{(13)}$ estudaram 1486 adenomas de 889 pacientes, e encontraram 64 pacientes $(7 \%)$ com pólipos adenomatosos com displasia de alto grau. Estes pacientes eram significativamente mais idosos do que os pacientes com displasia de baixo grau (67 \pm 11 anos versus $63 \pm 11$ anos, $P<0,01)$. Concordando com Kurome et al. ${ }^{(13)}$, O'Brien et al. ${ }^{(14)}$, analisaram 3371 pólipos de 1867 pacientes, e também observaram o aumento da idade associado com a displasia de alto grau.

Em nossa análise, a idade foi diferente estatisticamente em relação à prevalência dos adenomas quando comparados entre as faixas etárias $<40 \mathrm{e}$ de 40-49 anos com a faixa etária de 70-79 anos. Também houve diferença estatística quando dividimos as idades em dois grupos: $<50 \mathrm{e}=50$ anos $(P$ $<0,001)$. A comparação da idade dos pacientes com adenomas de alto grau com os de baixo grau não foi estatisticamente significativa. A não identificação da idade como fator de risco isolado para displasia de alto grau, pode estar associado ao número reduzido de pacientes com displasia de alto grau em nosso estudo, visto que a nossa frequência de pólipos de alto grau tenha sido semelhante aos dados da literatura $(13 \%)^{(4)}$.
A multiplicidade dos adenomas foi um achado controverso na literatura. No estudo de Kurome et $a l .{ }^{(13)}$, os pacientes com múltiplos adenomas apresentaram risco maior para displasia de alto grau. Entretanto, O'Brien et al. ${ }^{(14)}$ não identificaram este fator de risco. Foram analisados 28 pacientes com, ao total, 34 adenomas com displasia de alto grau. Entre estes, três pacientes tinham mais de um pólipo com displasia de alto grau. Em nossa análise não houve diferença estatisticamente significativa entre o número de adenomas e a displasia de alto grau.

Em relação ao tamanho, estudos anteriores evidenciaram que há relação entre a dimensão do adenoma e as características de patologia avançada. Verificou-se que quanto maior o tamanho da lesão $(=1$ $\mathrm{cm})$, maiores são as chances de displasia do pólipo ${ }^{(3,13 \text {, }}$ $17,19,20,21,22)$

Gschwantler et $_{\text {al. }}{ }^{(4)}$ tiveram 7590 adenomas incluídos no estudo. Entre estes, 6423 apresentaram displasia de baixo grau, $985(13 \%)$ displasia de alto grau e $182(2,4 \%)$ carcinoma invasivo. Entre seus resultados, o tamanho mostrou ser o mais importante fator de risco para características patológicas avançadas (advanced pathological features - APF). A porcentagem encontrada de adenomas com o APF foi de 3,4\%, $13,5 \%$ e $38,5 \%$ para os adenomas de diâmetro $<0,5$ $\mathrm{cm}, 0,5-1 \mathrm{~cm}$ e $>1 \mathrm{~cm}$, respectivamente. Concordando com achados prévios da literatura, nosso estudo colocou em evidência que adenomas com tamanho $=1 \mathrm{~cm}$ também estão relacionados como um fator de risco independente para displasia de alto grau. Encontramos 34 adenomas com displasia de alto grau e, entre estes, $64,7 \%$ eram $=1 \mathrm{~cm}$ comparados $\mathrm{com} 21,8 \%$ dos adenomas com displasia de baixo grau $(P<0,001)$.

Em alguns países, como no Japão, pólipos < $0,5 \mathrm{~cm}$ nem sempre são ressecados ${ }^{(13)}$ Em nosso estudo, entre os 326 adenomas, $8(4,6 \%)$ eram $<0,5 \mathrm{~cm}$ e tinham displasia de alto grau. Gschwantler et al. ${ }^{(4)}$ encontraram, entre os pólipos adenomatosos com displasia de alto grau, 3,4\% com diâmetro < 0,5 cm sugerindo que os mesmos sejam removidos quando possível.

Cânceres colorretais são mais frequentemente observados no cólon esquerdo do que no cólon direito ${ }^{(19,23)}$. Segundo Kurome et al. ${ }^{(13),}$ os resultados indicaram adenomas no cólon esquerdo como fator de risco para alto grau de displasia. Adenomas localizados no sigmóide e no reto apresentam uma maior tendência à displasia do que aqueles localizados mais proximalmente ${ }^{(14)}$. 
Alguns estudos na literatura encontraram uma porcentagem de adenomas com achados patológicos avançados mais elevada no lado esquerdo do cólon ${ }^{(14,}$ ${ }^{24,25)}$. Segundo Gschwantler et al. ${ }^{(4)}$, este fenômeno tem sido atribuído principalmente ao fato de que os adenomas no lado esquerdo do cólon são significativamente maiores e contém maior componente viloso do que no cólon direito.

Em nosso estudo encontramos aproximadamente metade dos adenomas com localização no reto e no sigmóide $(52,4 \%)$. Isto se assemelha à localização descrita na literatura dos cânceres colorretais, mas não encontramos relação significativa entre a localização dos adenomas e a displasia de alto grau.

Segundo o National Polyp Study, o qual analisou 3371 pólipos, foram encontrados fatores associados ao componente viloso e a displasia de alto grau ${ }^{(14)}$. Gschwantler et al. ${ }^{(4)}$ também mostraram uma correlação direta entre adenomas com patologia avançada e o componente viloso. Os nossos achados assemelham- se com aqueles encontrados na literatura, visto que, em nosso estudo, de 34 adenomas com displasia de alto grau, 29,4\% eram vilosos comparados com 4,1\% dos adenomas com displasia de baixo grau sendo estatisticamente significativo $(\mathrm{P}<0,001)$.

Não foi encontrada relação estatisticamente significativa entre a aparência macroscópica dos pólipos pediculados/subpediculados e sésseis, com pacientes com displasia de alto grau.

\section{CONCLUSÃO}

Concluímos que o tamanho $=1 \mathrm{~cm} \mathrm{dos}$ adenomas, assim como a presença do componente viloso nos pólipos adenomatosos, foram considerados fatores de risco independentes para displasia de alto grau. Portanto, determinar fatores que podem prever a existência de displasia de alto grau pode ajudar a identificar lesões clinicamente significativas que requerem maior atenção e tratamento imediato.

\begin{abstract}
Background: The study of polyps, especially adenomatous, is justified because of the straight correlation among them with the incidence of CRC. Objective: To analyze the risk factors for high-grade dysplasia of adenomatous polyps removed endoscopically. Methods: All consecutive colonoscopies performed by the Colorectal Department of the Hospital Nossa Senhora da Conceição, in Porto Alegre/RS, from January 2003 to December 2006, were evaluated. Patients diagnosed with adenomatous polyps were included. The relationship among tumors with high-grade dysplasia and the features of the patients and polyps were analyzed. Results: Among the 1,821 exams, 326 adenomas were detected in 208 patients. $51.4 \%$ of the patients were male; $69,2 \%$ had only one adenoma. A total of 28 patients $(13,5 \%)$ with 34 adenomas $(10,4 \%)$ with high-grade dysplasia were found. Among the tumors with high-grade dysplasia, $64.7 \%$ were $=1 \mathrm{~cm}, 61.8 \%$ were sessile, $29.4 \%$ were villous, and $70.6 \%$ were located in the left colon. Comparing the characteristics of the group of tumors of low grade dysplasia with those of high-grade, the size $=1 \mathrm{~cm}$ and villous component $(P<0,001)$ were statistically significant. Conclusion: Adenomas $=1 \mathrm{~cm}$ and dominance of villous component were considered independent risk factors for high-grade dysplasia.
\end{abstract}

Key words: Adenoma; Adenocarcinoma; Polyps; Colorectal neoplasms; Colonoscopy.

\section{REFERÊNCIAS}

1. Jemal A, Siegel R, Ward E, Hao Y, Xu J, Murray T, et al. Cancer Statistics, 2008. CA Cancer J Clin. 2008; 58: p 71 - 96.

2. Instituto Nacional do Câncer. Estimativas 2008: Incidência de Câncer no Brasil. INCA, 2007. Disponível em: http:// www.inca.gov.br

3. Park DH, Kim HS, Kim TI, Kim YH, Park DI, Kim HJ, et al. Clinicopathologic characteristics and malignant potential of colorectal flat neoplasia compared with that of polypoid neoplasia. Dis Colon Rectum. 2008 Jan; 51(1): p 43-49.

4. Gschwantler M, Kriwanek S, Langner E, Goritzer B, SchrutkaKolbl C, Brownstone E, et al. High-grade dysplasia and invasive carcinoma in colorectal adenomas: a multivariate analysis of the impact of adenoma and patient characteristics. Eur J Gastroenterol Hepatol 2002; 14: p 183-188.

5. Neugut AI, Jacobson JS, De Vivo I. Epidemiology of colorectal adenomatous polyps. Cancer Epidemiol Biomarkers Prev 1993; 2: p 159-176.

6. Leslie A, Carey FA, Pratt NR, Steele RJ. The colorectal adenoma-carcinoma sequence. Br J Surg. 2002 Jul; 89(7): p 845-860.

7. Mandel JS, Church TR, Bond JH, Ederer F, Geisser MS, Mongin SJ, et al. The effect of fecal occult-blood screening on the incidence of colorectal cancer. N Engl J Med. 2000; 343: p 1603-1607.

8. Harb-Gama A. Colorectal cancer- The importance of prevention. Arq Gastroenterol 2005; 42(1): p 2-3. 
9. Levin B, Lieberman DA, McFarland B, Andrews KS, Brooks D, Bond J, et al. Screening and Surveillance for the Early Detection of Colorectal Cancer and Adenomatous Polyps, 2008: A Joint Guideline From the American Cancer Society, the US Multi-Society Task Force on Colorectal Cancer, and the American College of Radiology. Gastroenterology. 2008 May;134(5): p 1570-1595.

10. Kronborg O, Fenger C, Olsen J, Jorgensen OD, Sondergaard $\mathrm{O}$. Randomised study of screening for colorectal cancer with faecaloccult- blood test. Lancet 1996;348: p 1467-1471.

11. Hardcastle JD, Chamberlain JO, Robinson MH, Moss SM, Amar SS, Balfour TW, et al. Randomised controlled trial of faecal-occultblood screening for colorectal cancer. Lancet 1996;348: p 1472-1477.

12. Rossi BM, Nagawawa WT, Ferreira FO, Aguiar Jr. S, Lopes A. Classificação morfológica de pólipos colorretais. In: Nakagava W, Ferreira F. Câncer de Cólon, Reto e Ânus. São Paulo: Lemar e Tecmed; 2004. p 175-205.

13. Kurome M, Kato J, Nawa T, Fujimoto T, Yamamoto H, Shiode $\mathrm{J}$, et al. Risk factors for high-grade dysplasia or carcinoma in colorectal adenoma cases treated with endoscopic polypectomy. Eur J Gastroenterol Hepatol 2008. 20:111117.

14. O'Brien MJ, Winawer SJ, Zauber AG, Gottlieb LS, Sternberg SS, Diaz B, et al. The National Polyp Study. Patient and polyp characteristics associated with high-grade dysplasia in colorectal adenomas. Gastroenterology 1990; 98: p 371-379.

15. Winawer SJ, Zauber AG, Ho MN, O'Brien MJ, Gottlieb LS, Sternberg SS, et al. Prevention of colorectal cancer by colonoscopic polypectomy. The National Polyp Study Workgroup. N Engl J Med 1993;329: p 1977-1981.

16. Regula J, Rupinski M, Kraszewska E, Polkowski M, Pachlewski J, Orlowska J, et al. Colonoscopy in colorectalcancer screening for detection of advanced neoplasia. N Engl J Med 2006; 355: p 1863-1872.

17. Lieberman DA, Weiss DG, Bond JH, Ahnen DJ, Garewal H, Chejfec G. Use of colonoscopy to screen asymptomatic adults for colorectal cancer. Veterans Affairs Cooperative Study Group 380. N Engl J Med 2000; 343: p 162-168.

18. Imperiale TF, Wagner DR, Lin CY, Larkin GN, Rogge JD, Ransohoff DF. Results of screening colonoscopy among persons 40-49 years of age. N Engl J Med 2002; 346: p 1781-1785.

19. Strul H, Kariv R, Leshno M, Halak A, Jakubowicz M, Santo $\mathrm{M}$, et al. The prevalence rate and anatomic location of colorectal adenoma and cancer detected by colonoscopy in average-risk individuals aged 40-80 years. Am J Gastroenterol 2006; 101: p 255-262.

20. Schoenfeld P, Cash B, Flood A, Dobhan R, Eastone J, Coyle $\mathrm{W}$, et al. Colonoscopic screening of average-risk women for colorectal neoplasia. N Engl J Med 2005; 352: p 2061-2068.

21. McCashland TM, Brand R, Lyden E, de Garmo P. Gender differences in colorectal polyps and tumors. Am J Gastroenterol 2001; 96: p 882-886.

22. Yamamoto M, Mine H, Kusumoto H, Maehara Y, Sugimachi K. Polyps with different grades of dysplasia and their distribution in the colorectum. Hepatogastroenterology 2004; 51: p 121-123.

23. Nusko G, Mansmann U, Partzsch U, Altendorf-Hofmann A, Groitl H, Wittekind C, et al. Invasive carcinoma in colorectal adenomas: multivariate analysis of patient and adenoma characteristics. Endoscopy 1997; 29: p 626-631.

24. Williams AR, Balasooriya BAW, Day DW. Polyps and cancer of the large bowel: a necropsy study in Liverpool. Gut 1982; 23: p 835-842.

25. Matek W, Hermanek P, Demling P. Is the adenoma-carcinoma sequence contradicted by the differing location of colorectal adenomas and carcinomas? Endoscopy 1986; 18: p 17-19.

\section{Endereço para correspondência: \\ JULIA SCHMIDT SILVA \\ Rua Guajuvira, 429, bairro Guarani. \\ Novo Hamburgo, Rio Grande do Sul. \\ CEP: $93520-200$ \\ E-mail: jumedicina@yahoo.com.br}

